03

\title{
Оптические характеристики неоднородного слоя титаната бария, легированного европием
}

\author{
(c) Н.И. Стаськов ${ }^{1}$, А.Б. Сотский ${ }^{1}$, Л.И. Сотская ${ }^{2}$, Н.В. Гапоненко ${ }^{3}$, Е.И. Лашковская ${ }^{3}$, А.Н. Петлицкий ${ }^{4}$, \\ A.А. Козлов ${ }^{5}$ \\ ${ }^{1}$ Могилевсктй государственный университет им. А.А. Кулешова, \\ 212022 Могилев, Республика Беларусь \\ ${ }^{2}$ Белорусско-Российский университет, \\ 212000 Могилев, Республика Беларусь \\ ${ }^{3}$ Белорусский государственный университет информатики и радиоэлектроники, \\ 220013 Минск, Республика Беларусь \\ ${ }^{4}$ Белмикросистемы НТЦ Филиал ОАО Интеграл, \\ 220108 Минск, Республика Беларусь \\ ${ }^{5}$ Пермский государственный национальный исследовательский университет, \\ 614990 Пермь, Россия \\ e-mail: ni_staskov@mail.ru
}

Поступила в редакцию 26.11.2020 г.

В окончательной редакции 24.12.2020 г.

Принята к публикации 26.12.2020 г.

\begin{abstract}
Разработан алгоритм решения обратной задачи многоугловой спектрофотометрии неоднородной поглощающей пленки, основанный на полиномиальных представлениях плотности материала пленки и его комплексного коэффициента рефракции. Алгоритм применен для определения спектральных и пространственных распределений показателей преломления и поглощения пленки титаната бария, легированного европием. Пленка нанесена по золь-гель-технологии на слой диоксида кремния, находящийся на кремниевой подложке. Корректность полученного решения проверена сравнением рассчитанных и измеренных спектров эллипсометрических углов и спектров отражательной способности структуры при нормальном падении света. Легирование европием титаната бария приводит к уменьшению длины волны, соответствующей краю полосы собственного поглощения, увеличению ширины запрещенной зоны материала в приповерхностном слое пленки и снижению концентрации европия вглубь пленки.
\end{abstract}

Ключевые слова: многоугловая спектрофотометрия, эллипсометрия, неоднородный поглощающий слой, оптические характеристики титаната бария, легированного европием.

DOI: $10.21883 /$ OS.2021.04.50782.287-20

\section{Введение}

Известно, что оптические характеристики планарных структур определяются методами их получения [1]. Поэтому использование при проектировании таких структур литературных данных о показателях преломления и поглощения однородных объемных материалов может приводить к ошибочным результатам, в особенности если технологический процесс приводит к образованию неоднородных слоев. В результате возникает проблема определения комплексного показателя преломления слоя $n(\lambda, y)$, где $\lambda$ - длина волны, $y$ - координата, отсчитываемая от поверхности слоя, которая рассматривается в настоящей работе.

В последнее время большое внимание уделяется зольгель-синтезу пленок титаната бария $\left(\mathrm{BaTiO}_{3}\right)$, которые имеют высокое значение показателя преломления [2] и спонтанную поляризацию, для применений в электронике и нанофотонике [3]. Легирование $\mathrm{BaTiO}_{3}$ лантаноидами приводит к интенсивной люминесценции в широком температурном диапазоне [4,5]. Наряду с этим наблюдали изменение оптических характеристик экспериментальных образцов фотонных кристаллов, в которых диоксид кремния $\left(\mathrm{SiO}_{2}\right)$ пропитывался титанатами $\mathrm{Ba}, \mathrm{Sr}$ или $\mathrm{Pb}$, при изменении температуры [6]. Пленки диоксида кремния в сочетании с пленками с более высоким показателем преломления применяются в качестве обкладок планарного волновода, компонентов брэгговских зеркал и микрорезонаторов [7,8]. Дальнейшее развитие технологии формирования структур с титанатом бария требует изучения оптических свойств золь-гель-пленок $\mathrm{BaTiO}_{3}$, содержащих люминесцирующие ионы и контактирующих с диоксидом кремния.

Формирование пленок титаната бария при определенном составе геля предполагает центрифугирование, сушку и отжиг. Снимки РЭМ показывают [2] превращение мелкозернистой аморфно-кристаллической структуры пленки $\mathrm{BaTiO}_{3}$, отожженной при $450^{\circ} \mathrm{C}$, в крупнозернистую структуру пленок, отожженных при 600 и $700^{\circ} \mathrm{C}$. В то же время не исключается неоднородность пленок по толщине. В работе [9] отмечается, что точные решения задачи об отражении света от неоднородного 
поверхностного слоя найдены для нескольких частных случаев профиля показателя преломления. В литературе чаще всего используется трехслойная электродинамическая модель, в которой принимается, что однородные золь-гель-пленки имеют оболочку в виде однородных переходных слоев с воздухом и подложкой [2]. Однако практика ее применения показала, что комплексные показатели преломления $n(\lambda)$ слоев, восстанавливаемые с использованием этой модели методами спектрофотометрии и спектральной эллипсометрии, существенно различаются. Возможно, это является следствием влияния непрерывной функции $n(\lambda, y)$ золь-гель-пленки на измеряемые параметры в разных по чувствительности оптических методах.

В настоящей работе методами спектрофотометрии и спектральной эллипсометрии исследована пленка титаната бария, легированного европием (BaTiO:Eu), которая нанесена по золь-гель-технологии на пленку диоксида кремния на кремниевой подложке. Предпринятые нами попытки определить оптические характеристики структуры с помощью стандартных компьютерных программ, использующих дисперсионные модели однородных слоев, прилагаемых к измерительным установкам Photon RT и UVISEL 2, не привели к удовлетворительному совпадению теории и эксперимента. Это явилось стимулом к разработке нового алгоритма решения обратной задачи многоугловой спектрофотометрии, основанного на модели неоднородной поглощающей пленки. Его использование для обработки экспериментальных спектров отражательной способности структуры для волн $s$ - и $p$-поляризации, измеренных при четырех углах падения света на структуру, обеспечило близкое согласие теории и эксперимента и позволило определить оптические характеристики структуры. Расчетом и сравнением с экспериментом спектра отражательной способности структуры при нормальном падении света, а также расчетом и сравнением с экспериментом спектров эллипсометрических углов при нескольких углах падения подтверждена корректность восстановленных вещественной и мнимой частей функции $n(\lambda, y)$.

\section{Теория}

В [10] был предложен метод решения обратной задачи отражательной спектрофотометрии неоднородного слоя, основанный на представлении его диэлектрической проницаемости моделью Лорентц-Лоренца. Однако алгоритм обработки экспериментальных спектрофотометрических данных для исследуемой структуры, взятый из [10], так же, как и отмеченные выше подходы, привел к значительному расхождению теории и эксперимента. Мы связали этот факт с использованием в названном алгоритме приближения $\operatorname{Im} n(\lambda, y)=0$. Ниже предлагается более общая схема решения обратной оптической задачи спектрофотометрии неоднородного слоя, которая свободна от указанного приближения.
Пусть слой занимает область $-d \leq y \leq 0$. В области $y<-d$ находится подложка с известным показателем преломления $n_{s}(\lambda)$. Плоская волна $s$ - либо $p$ поляризации падает на слой под углом $\theta$ из области $y>0$, занятой воздухом. Измеряются спектры энергетических коэффициентов отражения $R_{\kappa}$, где $\kappa$ пробегает $K=2 N M$ значений (множитель 2 учитывает $s$ - и $p$ волны, $N$ - число углов падения, $M$ - число использованных длин волн).

Расчет значений $R_{\kappa}=\left|r_{\kappa}\right|^{2}$, где $r_{\kappa}$ - амплитудный коэффициент отражения плоской волны от слоя, проводим методом стратификации. В нем неоднородный слой заменяется последовательностью $m$ однородных слоев. Толщина слоев $\Delta y=d / m$, диэлектрическая проницаемость $j$-го слоя $\varepsilon_{j}=n^{2}[\lambda,-d+\Delta y(j-0.5)](j=\overline{1, m}$. Для волн $s$-поляризации

$$
r_{\kappa}=\left(i k_{y} \psi_{m+1}-\psi_{m+1}^{\prime}\right)\left(i k_{y} \psi_{m+1}+\psi_{m+1}^{\prime}\right)^{-1}
$$

где $k_{y}=k_{0} \sqrt{\varepsilon_{a}-\beta^{2}}, \varepsilon_{a}=n_{a}^{2}, k_{0}=2 \pi \lambda^{-1} \beta=n_{a} \sin \theta$, $n_{a}$ - показатель преломления воздуха, $\psi_{m+1}, \psi_{m+1}^{\prime}$ рассчитываются по рекуррентным формулам

$$
\begin{aligned}
& \psi_{j+1}=\psi_{j} \cos \left(\sigma_{j} \Delta y\right)+\psi_{j}^{\prime} \sin \left(\sigma_{j} \Delta y\right) \sigma_{j}^{-1}, \\
& \psi_{j+1}^{\prime}=-\psi_{j} \sigma_{j} \sin \left(\sigma_{j} \Delta y\right)+\psi_{j}^{\prime} \cos \left(\sigma_{j} \Delta y\right),
\end{aligned}
$$

где $\quad \psi_{1}=1, \quad \psi_{1}^{\prime}=-\sigma_{s}, \quad \sigma_{j}=k_{0} \sqrt{\varepsilon_{j}-\beta^{2}}$, $\sigma_{s}=k_{0} \sqrt{\varepsilon_{s}-\beta^{2}}, \quad \varepsilon_{s}=n_{s}^{2}$. Для волн $p$-поляризации $\psi_{1}=1, \psi_{1}^{\prime}=i \sigma_{s} \varepsilon_{s}^{-1}$,

$$
\begin{gathered}
r_{\kappa}=\left(i k_{y} \psi_{m+1}-n_{a}^{2} \psi_{m+1}^{\prime}\right)\left(i k_{y} \psi_{m+1}+n_{a}^{2} \psi_{m+1}^{\prime}\right)^{-1}, \\
\psi_{j+1}=\psi_{j} \cos \left(\sigma_{j} \Delta y\right)+\psi_{j}^{\prime} \varepsilon_{j} \sin \left(\sigma_{j k} \Delta\right) \sigma_{j}^{-1}, \\
\psi_{j+1}^{\prime}=-\psi_{j} \sigma_{j} \varepsilon_{j}^{-1} \sin \left(\sigma_{j} \Delta y\right)+\psi_{j}^{\prime} \cos \left(\sigma_{j k} \Delta y\right) .
\end{gathered}
$$

Модель Лорентц-Лоренца подразумевает задание комплексной диэлектрической проницаемости неоднородного слоя в виде [10]

$$
\varepsilon(\lambda, y)=[1+2 \rho(\lambda) f(\eta)][1-\rho(\lambda) f(\eta)]^{-1},
$$

где $\eta=y d^{-1}, d$ - толщина слоя, $\rho(\lambda)$ и $f(\eta)-$ комплексный коэффициент рефракции и вещественная функция плотности материала слоя. Поскольку $\rho(\lambda)$ и $f(\eta)$ входят в (3) в виде произведения, функция $f(\eta)$ определена с точностью до произвольного множителя. Далее ее нормировка выбрана в соответствии с условием

$$
\int_{-d}^{0} f(\eta) d \eta=1
$$

Функции $\rho(\lambda)$ и $f(y)$ ищем в форме полиномов

$$
\rho(\lambda)=\sum_{j=1}^{L L} \rho_{j} P L(j, \lambda), \quad f(\eta)=1+\sum_{j=1}^{L Y} f_{J} P Y(j, \eta),
$$


где $\quad P L(j, \lambda)=\left[(\lambda-\bar{\lambda})(B-\bar{\lambda})^{-1}\right]^{j-1}, \quad P Y(j, \eta)=\eta^{j}+$ $(-1)^{j+1}(j+1)^{-1}, \bar{\lambda}=0.5(A+B), A$ и $B-$ границы спектрального диапазона, $L L-1$ и $L Y-$ порядки полиномов, $d, \rho_{j}, f_{j}$ - параметры, подлежащие определению. Они находятся методом наименьших квадратов с целевой функцией

$$
I\left(p_{1}, \ldots, p_{l}\right)=\sum_{\kappa=1}^{K}\left(R_{\kappa}=\left|r_{\kappa}\left(p_{1}, \ldots, p_{l}\right)\right|^{2}\right)^{2}
$$

от $2 L L+L Y+1$ вещественных переменных $p_{1}, \ldots, p_{l}$. Здесь $\quad l=2 L L+L Y+1 \ll K, \quad p_{1}=d, \quad p_{j}=\operatorname{Re} \rho_{j-1}$ при $j=2, \ldots, L L+1, \rho_{J}=\operatorname{Im} \rho_{j-L L-1}, j=L L+2, \ldots$, $2 L L+2, \quad p_{J}=f_{j-2 L L-1}, \quad j=2 L L+2, \ldots, l, \quad R_{\kappa}-$ эксперимент. Для отыскания минимума функции (4) использована итерационная схема [10]

$$
\begin{gathered}
p_{j}^{(k+1)}=p_{j}^{(k)}+\sum_{i=1}^{l}\left(\mathbf{Q}^{-1}\right)_{j i}(\mathbf{B})_{i}, \\
(\mathbf{Q})_{i j}=\sum_{, \kappa=1}^{K} \operatorname{Re}\left(r_{\kappa}^{*} \frac{\partial r_{\kappa}}{\partial p_{i}}\right) \operatorname{Re}\left(r_{\kappa}^{*} \frac{\partial r_{\kappa}}{\partial p_{j}}\right), \\
(\mathbf{B})_{i}=0.5 \sum_{\kappa=1}^{K}\left(R_{\kappa}-\left|r_{\kappa}\right|^{2}\right) \operatorname{Re}\left(r_{\kappa}^{*} \frac{\partial r_{\kappa}}{\partial p_{i}}\right),
\end{gathered}
$$

где $k$ - номер итерации, матрица $\mathbf{Q}$ и вектор В соответствуют $p_{j}=p_{j}^{(k)}$. Применение этой схемы позволило удовлетворительно описать спектрофотометрические эксперименты для структуры $\mathrm{BaTiO}_{3}: \mathrm{Eu}-\mathrm{SiO}_{2}-\mathrm{cSi}$ и определить ее оптическую функцию $n(\lambda, y)$.

\section{Эксперимент}

На четырехдюймовой пластине кристаллического кремния толщиной $395 \mu \mathrm{m}$ была выращена пленка оксида кремния методом влажного термического оксидирования в установке PEO-601 компании ATV. Процесс окисления происходит при температуре $1100^{\circ} \mathrm{C}$ в течение $13 \mathrm{~h}$. Для получения пленки $\mathrm{BaTiO}_{3}: \mathrm{Eu}$ использовали золь, приготовленный по методике [5], в котором концентрации титаната бария $\left(\mathrm{BaTiO}_{3}\right)$ и оксида европия $\left(\mathrm{Eu}_{2} \mathrm{O}_{3}\right)$ составляли соответственно 56.6 и $2.209 \mathrm{mg} / \mathrm{ml}$. Золь наносили центрифугированием при скорости 1600 turn/min в течение $30 \mathrm{~s}$ на пленку оксида кремния, которая для лучшей адгезии очищалась в растворе дихромата натрия и промывалась дистиллированной водой. Затем образец сушился при температуpe $150^{\circ} \mathrm{C}$ в течение $10 \mathrm{~min}$ и отжигался при температуpe $450^{\circ} \mathrm{C}$ в течение $30 \mathrm{~min}$.

$\mathrm{O}$ морфологии пленки $\mathrm{BaTiO}_{3}: \mathrm{Eu}$ на слое $\mathrm{SiO}_{2}$ позволяет судить снимок его скола, полученный на электронном микроскопе (РЭМ) Hitachi S-4800 (рис. 1).

Снимок позволяет оценить толщину слоя $\mathrm{BaTiO}_{3}: \mathrm{Eu}$ значением $\sim 76 \mathrm{~nm}$, а толщину подслоя $\mathrm{SiO}_{2}-$ значени$\mathrm{eM} \sim 560 \mathrm{~nm}$.

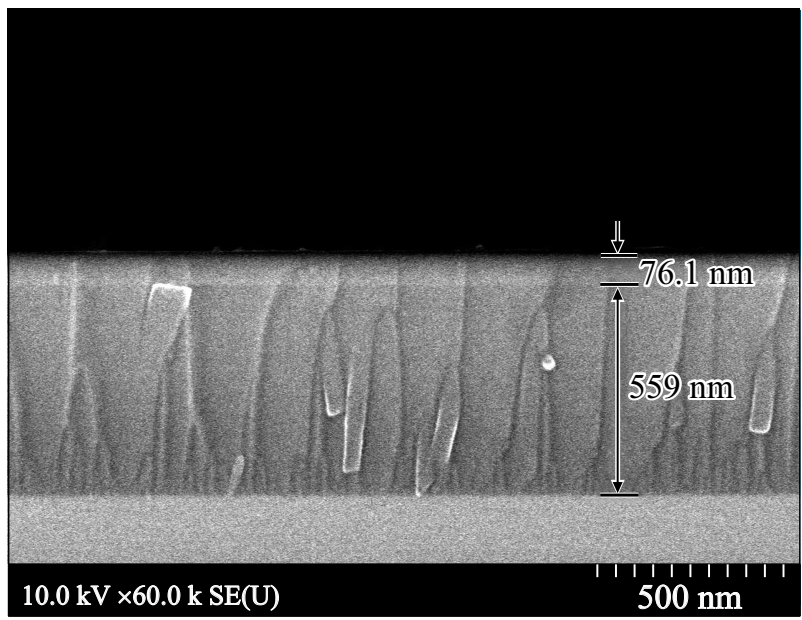

Pис. 1. РЭМ-снимок структуры $\mathrm{BaTiO}_{3}: \mathrm{Eu}-\mathrm{SiO}_{2}-\mathrm{cSi}$.

Исследование оптических свойств рассматриваемой структуры выполнено на спектрофотометре Photon RT (ЭссентОптикс, Беларусь), микроспектрофотометpe MPV-SP (Leico, Германия) и спектроэллипсометре UVISEL2 (HORIBA, Франция). На спектрофотометре Photon RT измерены спектры $R_{\kappa}$ для $s$ - и $p$-волн в области от 200 до $950 \mathrm{~nm}$ с шагом $2 \mathrm{~nm}$ при углах падения $\theta=10,20,50,70^{\circ}(K=2952)$. По этим данным рассчитаны спектры действительной и мнимой частей функции $n(\lambda, y)$ структуры $\mathrm{BaTiO}_{3}: \mathrm{Eu}-\mathrm{SiO}_{2}-\mathrm{cSi}$. На рис. 2, a представлены измеренные спектры $R_{s}$ ( 1 Photon RT, $\left.\theta=70^{\circ}\right)$ и $R\left(3-\mathrm{MPV}-\mathrm{SP}, \theta=0^{\circ}\right)$. Pacчеты соответствующих спектров 2 и 4 выполнены с использованием функции $\eta(\lambda, y)$. На рис. $2, b$ представлены измеренные $\left(1,3\right.$ - UVISEL2, $\left.\theta=70^{\circ}\right)$ и рассчитанные $(2,4)$ с использованием функции $n(\lambda, y)$ спектры функций эллипсометрических углов $\tan (\psi)(1,2)$ и $\cos (\Delta)(3,4)$. Приведенные данные позволяют судить, с одной стороны, о соответствии теории и эксперимента (невязка не превышает 0.01) на разных оптических приборах, а с другой стороны, являются доказательством корректности численных расчетов (рис. 3) функции $n(\lambda, y)$.

\section{Оптические характеристики слоя}

Дисперсионные свойства материалов слоев иллюстрирует рис. 3 . Он соответствует диапазону $d \leq y \leq 0$, где найденное значение $d=p_{1}=610 \mathrm{~nm}$. Дисперсия объемного кристаллического $\mathrm{BaTiO}_{3}$ [11] (штриховая кривая) существенно отличается от дисперсии материала слоя $\mathrm{BaTiO}_{3}: \mathrm{Eu}$. Вблизи границы раздела воздух $-\mathrm{BaTiO}_{3}: \mathrm{Eu}$ $(y=-14 \mathrm{~nm})$ показатель преломления слоя в видимой спектральной области близок к показателю преломления чистой золь-гель-пленки $\mathrm{BaTiO}_{3}$, которая была получена при температуре отжига $450^{\circ} \mathrm{C}$ [2]. Отличие наблюдается в УФ области, где расположена полоса собствен- 

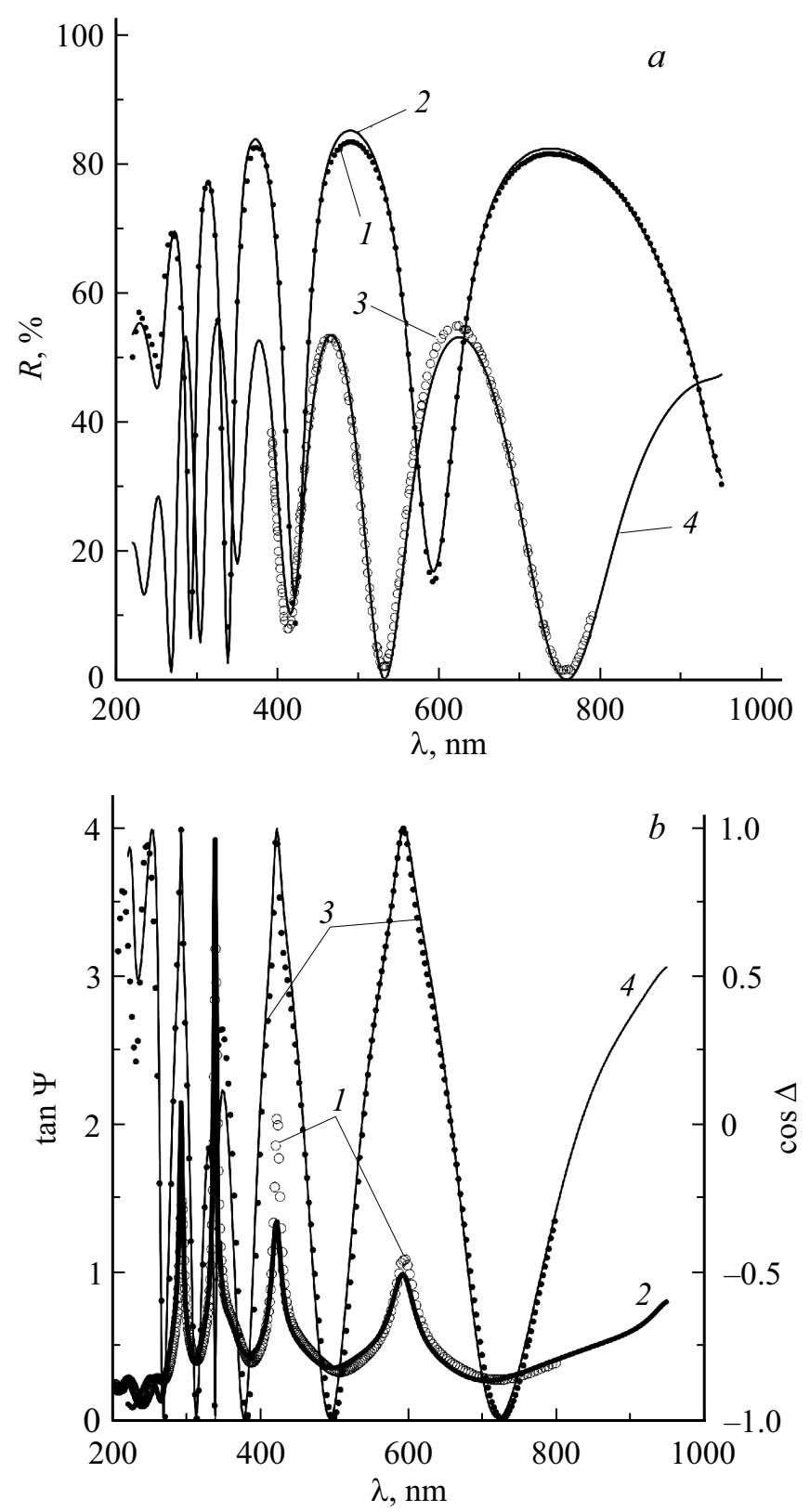

Рис. 2. Отражательные способности $(a)$ и эллипсометрические параметры $(b)$ структуры $\mathrm{BaTiO}_{3}: \mathrm{Eu}-\mathrm{SiO}_{2}-\mathrm{cSi}$. 1,3 эксперимент; 2, 4 - теория.

ного поглощения титаната бария. Это указывает на изменении ширины запрещенной зоны при легировании $\mathrm{BaTiO}_{3}$ европием. Уменьшение длины волны, которая характеризует край полосы поглощения на спектрах $|\operatorname{Im} n|$, свидетельствует об увеличении ширины запрещенной зоны материала неоднородного слоя с удалением от поверхности. На глубине $y=-415 \mathrm{~nm}$ показатель преломления неоднородного слоя в видимой спектральной области близок к показателю преломления термически окисленного слоя $\mathrm{SiO}_{2}$ [12]. Золь-гель-пленки из чистого $\mathrm{BaTiO}_{3}$, отожженные при $450^{\circ} \mathrm{C}$, прозрачны $(|\operatorname{Im} n|=0)$ в видимой области спектра [2]. Легирование европием титаната бария нарушает прозрачность этих пленок. Наиболее поглощающим оказывается приповерхностный слой $(y=-14 \mathrm{~nm})$. С удалением от поверхности показатель поглощения неоднородного слоя уменьшается.

Функция $f(y)$ на рис. 4, $a$, характеризующая распределение плотности материала по толщине слоя, согласуется с данными рис. 1. С удалением от поверхности $(y=0)$ плотность неоднородного слоя уменьшается, затем достигает максимума и снова уменьшается. Такое поведение функции $f(y)$ можно объяснить шероховатостью поверхности слоя $\mathrm{BaTiO}_{3}: \mathrm{Eu}$, частичным разруше-
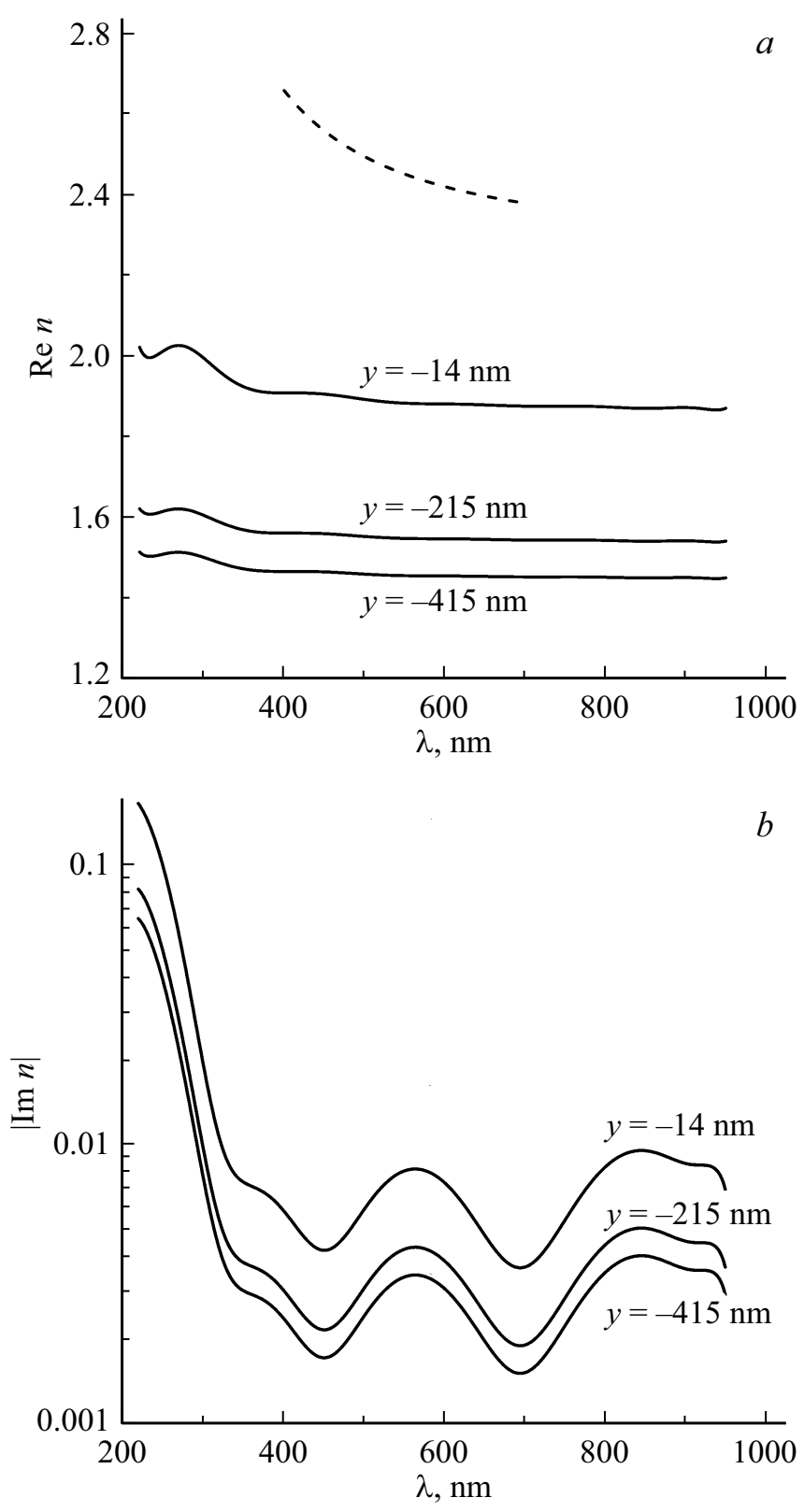

Рис. 3. Сплошные кривые - восстановленные спектры вещественной $(a)$ и мнимой $(b)$ частей показателя преломления $\mathrm{BaTiO}_{3}: \mathrm{Eu}-\mathrm{SiO}_{2}$ при указанных $y$. Штриховая кривая известный из литературы спектр показателя преломления кристалла $\mathrm{BaTiO}_{3}$ [11]. 

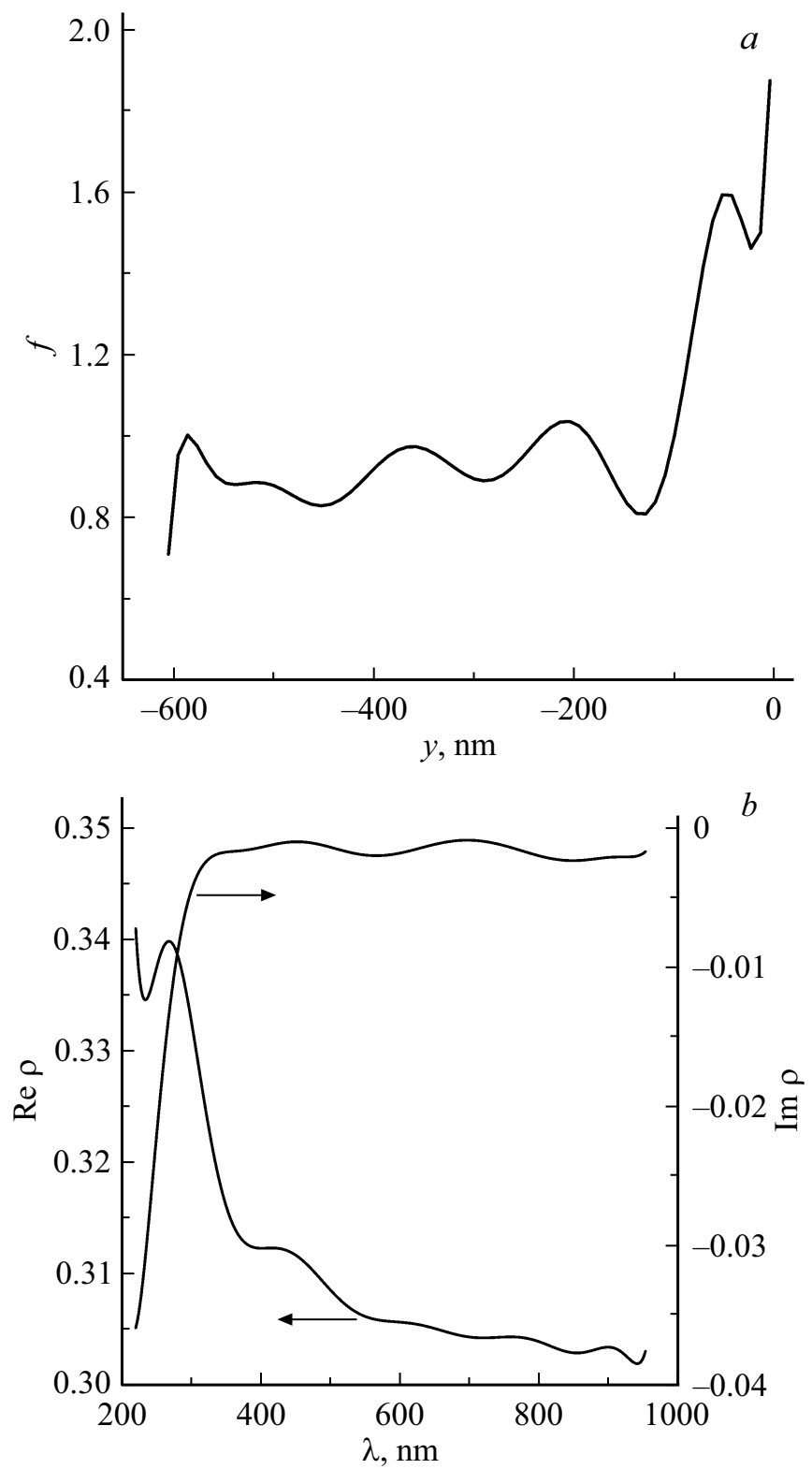

Рис. 4. Восстановленные функция плотности $(a)$ и коэффициент рефракции $(b)$ неоднородного слоя $\mathrm{BaTiO}_{3}: \mathrm{Eu}-\mathrm{SiO}_{2}$.

нием приповерхностного слоя при химической очистке поверхности пленки диоксида кремния и уменьшением концентрации европия по толщине слоя при удалении от его поверхности.

\section{Заключение}

Предложен метод обработки спектров отражательной способности неоднородного поглощающего слоя, измеряемых для волн $s$ - и $p$-поляризаций при нескольких углах падения света на слой, в котором для представления показателя преломления слоя используется модель Лорентц-Лоренца с полиномиальными представлениями функции плотности материала слоя и комплексного коэффициента рефракции.

Вычислительные эксперименты по расчету названных спектров для различных неоднородных поглощающих слоев с последующим определением их параметров принятым методом показали, что он эффективен в случае достаточно толстых слоев, спектры отражательных способностей которых имеют как минимум один интерференционный экстремум на экспериментальном интервале длин волн. Это ограничение аналогично тому, которое было отмечено в [10] при исследовании неоднородных прозрачных слоев. Его причиной является естественное снижение чувствительности спектров отражения к деталям профиля показателя преломления слоя по мере уменьшения его толщины [10].

Метод с успехом применен к исследованию зольгель-пленки $\mathrm{BaTiO}_{3}: \mathrm{Eu}$, расположенной на термически окисленном кремнии, спектры отражательной способности которой имеют около 10 интерференционных экстремумов (рис. 2). Определены геометрические и оптические характеристики пленки. Установлено, что легирование европием титаната бария нарушает прозрачность пленок. Наиболее поглощающим оказывается приповерхностный слой (толщиной около $10 \mathrm{~nm}$ ) с повышенным содержанием европия. В видимой спектральной области показатель преломления этого слоя близок к показателю преломления чистой золь-гельпленки $\mathrm{BaTiO}_{3}$, отожженной при $450^{\circ} \mathrm{C}$. С удалением от поверхности показатели преломления и поглощения неоднородного слоя уменьшаются и плавно переходят к значениям, характерным для термического диоксида кремния.

Возможное изменение концентрации лантаноидов по толщине пленки, формируемой золь-гель-методом, необходимо учитывать ввиду ион-ионного взаимодействия лантаноидов, которое может оказать влияние на их спектрально-люминесцентные свойства за счет эффектов ап-конверсии и кросс-релаксации [13].

\section{Финансирование работы}

Работа выполнена в рамках ГПНИ РБ „Фотоника, опто- и микроэлектроника 3.5.10. Исследование методами спектроэллипсометрии и спектрофотометрии фазового состава, геометрических и оптических дисперсионных характеристик многослойных нанометровых гетероструктур на основе диэлектриков, полупроводников и металлов“ и „Фотоника, опто- и микроэлектроника 1.2.03. Разработка теории методов оптического контроля наноразмерных тонкопленочных структур“.

\section{Конфликт интересов}

Авторы заявляют, что у них нет конфликта интересов. 


\section{Список литературы}

[1] Tikhonravov A.V., Trubetskov M.K., Amotchkina T.V., DeBell G., Pervak V., Krasilnikova Sytchkova A., Grilli M.L., Ristau D. // Applied Optics. 2011. V. 50. N 9. P. C75. doi 10.1364/AO.50.000C75

[2] Стаськов Н.И., Сотский А. Б., Михеев С.С., Гапоненко Н.В., Холов П.А., Райченок Т.Ф. // ЖПС. 2020. Т. 87. № 6. C. 918.

[3] Karvounis A., Timpu F., Vogler-Neuling V.V., Savo R., Grange $R$. // Advanced Optical Materials. 2020. P. 202001249. doi 10.1002/adom.202001249

[4] Strek W., Hreniak D., Boulon G., Guyot Y., Pazik R. // Optical Material. 2003. V. 24. N 1-2. P. 15. doi 10.1016/S09253467(03)00099-5

[5] Gaponenko N.V., Kholov P.A., Raichenok T.F., Prislopski S.Ya. // Optical Materials. 2019. V. 96. P. 109265. doi 10.1016/j.optmat.2019.109265

[6] Kim B.G., Parikh K.S., Ussery G., Zakhidov A., Baughman R.H., Yablonovitch E., Dunn B.S. // Appl. Phys. Lett. 2002. V. 81. P. 4440. doi 10.1063/1.1526163

[7] Polman A. // J. Appl. Phys. 1997. V. 82. N 1. P. 1. doi 10.1063/1.366265

[8] Vredenberg A.M., Hunt N.E.J., Schubert E.F., Jacobson D.C., Poate J.M., Zydzik G.J. // Phys. Rev. Lett. 1993. V. 71. P. 517. doi 10.1103/PhysRevLett.71.517

[9] Пшеницын В.И., Абаев М.И., Лызлов Н.Ю. Эллипсометрия в физико-химических исследованиях. Л.: Химия, 1986. $41 \mathrm{c}$.

[10] Сотский А.Б., Кривещкий К.Н., Парашков С.О., Сотская Л.И. // ЖПС. 2016. Т. 83. № $5 . \quad$ С. 809. doi 10.1007/s10812-016-0373-3

[11] Wemple S.H., Didomenico E.M., Camlibel I. // J. Phys. Chem. Solids. 1968. V. 29. P. 1797. doi 10.1016/00223697(68)90164-9

[12] Стаськов Н.И., Мухаммедмурадов А.А., Крекотень Н.А., Парашков С.О. // ЖПС. 2020. Т. 87. № 1. С. 122. doi 10.1007/s10812-020-00970-y

[13] Kenyon A.J. // Progress in Quantum Electronics. 2002. V. 26. P. 225-284. doi 10.1016/S0079-6727(02)00014-9 